\title{
CLASSIFICATION METHODS OF SKIN BURN IMAGES
}

\author{
Malini Suvarna ${ }^{1}$, Sivakumar $^{2}$ and U C Niranjan ${ }^{3}$ \\ ${ }^{1}$ Research Scholar, Dept. of E\&C, Atria Institute of Technology, Bangalore \\ maliniatria@gmail.com

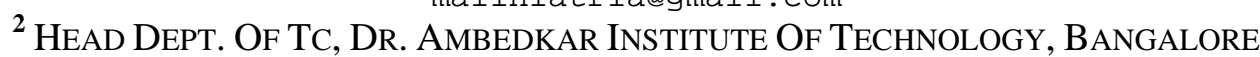 \\ sivabs2000@yahoo.co.uk \\ ${ }^{3}$ Director of Research, Manipal Dot ,Manipal \\ ucniranjaneyahoo.com
}

\begin{abstract}
In this paper, methods to automatically detect and categorize the severity of skin burn images using various classification techniques are compared and presented. A database comprising of skin burn images belonging to patients of diverse ethnicity, gender and age are considered. First the images are preprocessed and then classified utilizing the pattern recognition techniques: Template Matching (TM), K nearest neighbor classifier ( $k N N)$ and Support Vector Machine (SVM). The classifier is trained for different skin burn grades using pre-labeled images and optimized for the features chosen. This algorithm developed, works as an automatic skin burn wound analyzer and aids in the diagnosis of burn victims.
\end{abstract}

\section{KEYWORDS:}

$k N N, S V M$

\section{INTRODUCTION}

In the modern world accidents occur quite frequently. Many of them are serious and life threatening. Fire accidents are also quite serious and many a times have fatal endings. They need not be so if proper medical treatment is meted out expeditiously. But medical treatment might not be available at the site of the accident or in the necessity.

Even if medical facility were to be available it would be necessary for the doctor or medical attendant available to evaluate the injury before administering any treatment. Need less to say that a correct first treatment of my burn injury would go a long way in successful evaluation of the injury.[5]. In turn the first treatment would be dependent on the intensity and depth of the burn.

Burn units in medical facilities particularly in rural areas are restricted by its capital measurements. Other methods of burn injury imaging viz., thermal, infrared, ultraviolet \& radioactive isotope images are impractical. There are also know to have not too high accuracy levels. There fore these projects ventures into classifying burn images using colour characteristics of burn injury photographs and comparing them with references to decide on the first treatment. Lack of the burn specialist in a medical centre results in incorrect application of first treatment and the first treatment is very important for the proper evolution of wound. When a person meets with a burn accident the skin layers get affected. Doctors determine the degree of burns by examining which layer and organs are affected and suggest treatment. The work proposed here

DOI : 10.5121/ijcsit.2013.5109 
focuses on automating this process. The outcome of my research will facilitate medical health to burn patient as soon as possible. Many burn accidents in India have social causes such as bomb blasting, dowry death, honor killing etc. This research would contribute significantly to enhance the treatment given to burn patients. The proposed automatic wound analyzer would be useful at remote locations where medical expertise is not available. A digital camera is used to capture the burn images of the patient and the software developed would analyze the image. Using the outcome of this research, severity of injury can be estimated, and the depth of the injured tissue can be quantified. Ongoing experimental research on skin burn images is least. Thus, contribution to this field of research is not considerable. Contribution to this is done particularly by the researchers from Spain, belonging to Biomedical engineering group of Seville University. Though the work is of significant importance, very little research has been done.

The research scholars belonging to the biomedical engineering group of Seville University have made a study to find the effectiveness of telemedicine for plastic surgical applications. They have considered burn images for tele-diagnosis by capturing the burn image in a digital camera and compressing it for transmission through a communication media. Acha and Serrano have worked to classify the burn images using features of color and texture. This was done using CIE lab representation of color and vector quantizer algorithm analysis.

\section{BURN INJURIES}

II. Some burn injuries could be turned bad, some not too bad, some serious. How bad a burn injury is depends on certain factors. Viz.,

i) How the skin has burned

ii) How long the burn has fasted affecting the skin to varying depths.

iii) The location of the injury on the body also matter since different areas of the body differ in skin thickness,

water content as well as subcutaneous fat content as well as the member of blood vessels just

below the skin.[4]

\section{A. DIAGNOSING A BURN INJURY}

Irrespective of the location of the burn injury its seriousness depends on the area of skin burnt and the depth to which it has burnt. While different areas burnt could have different depths of burns depending on the exposure, normally the depth of burn is the maximum in the centre of each burn area. Although a series of tests can be conducted to determine the seriousness of the injury one cannot discount the assessment made by a skin specialist after done examination. The experts' opinion till date remains the most reliable evaluation of an injury. Evaluation is more difficult in cases where the pulmonary system is affected due to prolonged exposure to heat and inhabitation of smoke while flash burns cause injury to face but do not hare the airways.

\section{CLASSIFICATION OF BURN IMAGES}

Skin is the largest organ of our body and it gets damaged predominantly during burn accidents. Skin accounts for $15 \%$ of the total weight of an adult human being. The basic functions of the skin are protection, sensation, and temperature regulation, synthesis of vitamin D. The principal components of the human skin are epidermis and dermis. Epidermis is the outer thinner part of the skin, while the dermis is the inner thick layer of connective tissue made of elastic fibers. Once the skin burn images are enhanced, next step is to label these images as a specialist does for the proper treatment. This forms the crucial part of this work. It has been mentioned earlier that this 
project venturous in to classification of burn injury images using colour characteristics of burn photographs. Based on the colour-its hue and intensity of shade, from centre to the periphery-the skin burn could be classified in to three categories.

\section{A. SUPERFICIAL BURN}

Superficial burn means injury to the epidermal layer of the skin which normally heals within 5 to 7 days. A common type of superficial burn is sunburn. Figure 3.1 is a example of superficial burn.

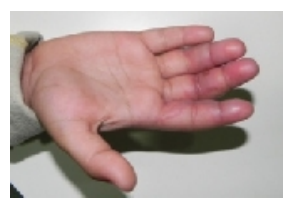

Fig 3.1 Superficial dermal burn

\section{B. PARTIAL THICKNESS BURN}

In the partial thickness burn the damage extends up to the dermis layer. As a result, the seriousness of a partial thickness burn depends on how much the dermis has been injured, these burns usually leave scars. Figure 3.2 is an example of partial thickness burns.

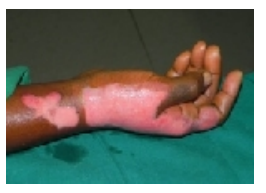

Fig 3.2 Partial thickness burn

\section{FULL THICKNESS BURN}

Full thickness burn destroys epidermis, dermis and subcutaneous layers of skin, with no sensation. Full thickness burns require skin grafts. Figure 3.3 is an example of full thickness burns.

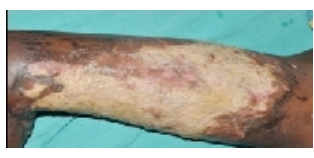

Fig 3.3 Full thickness burn

Table 3.4 Skin burn characteristics

\begin{tabular}{|l|l|l|l|}
\hline Type of Burn & Blistering & Appearance & $\begin{array}{l}\text { Pinprick } \\
\text { test }\end{array}$ \\
\hline $\begin{array}{l}\text { Superficial } \\
\text { dermal }\end{array}$ & Present & Bright red & $\begin{array}{l}\text { Sensitive } \\
\text { to pain }\end{array}$ \\
\hline $\begin{array}{l}\text { Partial } \\
\text { thickness }\end{array}$ & $\begin{array}{l}\text { Blisters } \\
\text { are } \\
\text { broken }\end{array}$ & $\begin{array}{l}\text { Cream } \\
\text { colored }\end{array}$ & $\begin{array}{l}\text { Dullness to } \\
\text { pain }\end{array}$ \\
\hline $\begin{array}{l}\text { Full } \\
\text { thickness }\end{array}$ & Absent & Gray/White & $\begin{array}{l}\text { No } \\
\text { sensation }\end{array}$ \\
\hline
\end{tabular}




\section{FEATURE EXTRACTION}

Feature selection is the very important factor while classifying the images into different categories. The selected features represent the characters of the images belonging to particular category. Since the color of the skin burn images differs based on the depth of the wound, the color features of each image are extracted and used for the classifier training $[1,2]$.

In this work, firstly the image is re-sized to $90 * 90$ pixels, secondly RGB space is converted into $\mathrm{L} * \mathrm{a} * \mathrm{~b} *$ color space. Photographs taken from different cameras are and bourn to have different pixels. All photographs first are resized to $90 * 90$ pixels for uniformly. Red (R), Green (G) and Blue (B) colours combine in different percentages to give a variety of hues. The RGB spaces the converted in to spaces bases on luminance components ' $a$ ' and ' $\mathrm{g}$ '. It is normally represented as $\mathrm{L} * \mathrm{a} * \mathrm{~b} *$ space.

After the pre-processing from RGB space to $\mathrm{L}^{*} \mathrm{a}^{*} \mathrm{~b}^{*}$ space is done, a plane refereed to as V1 chrominance of the $\mathrm{L}^{*} \mathrm{a} \mathrm{b}^{*}$ space is selected for feature extraction. Further a $90 * 90$ image is subdivided into $9 * 9$ blocks and the features like mean and $(2,1)$ th coefficient of Discrete Cosine Transform (DCT) function chosen to train classifiers. The two dimensional DCT equation is given by

$$
\begin{gathered}
X\left(k_{1} k_{2}\right)=\frac{4 \epsilon_{k 1} \in_{k 2}}{N^{2}} \sum_{n_{1}=0}^{N_{1}-1} \sum_{n_{2}=0}^{N_{2}-1} x\left(n_{1}, n_{2}\right) \cos \left(\frac{\pi\left(2 n_{1}+1\right) k_{1}}{2 N_{1}}\right) \cos \left(\frac{\pi\left(2 n_{2}+1\right) k_{2}}{2 N_{2}}\right) \\
\text { where }\left\{\begin{array}{c}
\epsilon_{\mathrm{k}}=\frac{1}{\sqrt{2}} \text { for } k=0 \\
=1 \text { otherwise }
\end{array}\right. \\
\text { and } k=0,1,2, \ldots \ldots, N
\end{gathered}
$$

\section{CLASSIFICATION METHODOLIGIES}

\section{A. TEMPLATE MATCHING METHOD}

In this method of classification comparison of data values is done. Templates belonging to different class of burn images are compared with the new samples. The sample image is classified into the class, whose template matches with the new sample with least error. Figure 4.1 illustrates the template matching method in which the features under selected region are compared with the previously stored values. The process is inherently such that it takes more time to process compute and throughout results when the sample size is more [5].

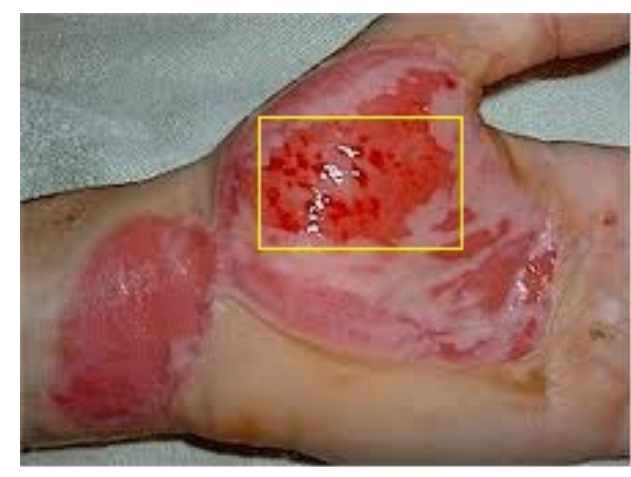

Figure 4.1: Template Matching Method 


\section{B. K-NEAREST NEIGHBOR CLASSIFIER}

In this method, the decision is made by examining the labels on the nearest neighbors and taking a vote. The kNN algorithm finds a group of $k$ objects in the training set that are closest to the test object, and bases the assignment of a label on the predominance of a particular class in this neighborhood. This addresses the issue that, in many data sets, it is unlikely that one object will exactly match another, as well as the fact that conflicting information about the class of an object may be provided by the objects closest to it. The method used to determine the class of the target object is based on the classes and distances of the $\mathrm{k}$ nearest neighbors $(8,9)$.

Given a training set $\mathrm{D}$ and a test object $\mathrm{z}$, which is a vector of attribute values and has an unknown class label, the algorithm computes the Euclidean distance between $\mathrm{z}$ and all the training objects to determine its nearest neighbor list. It then assigns a class to $\mathrm{z}$ by taking the class of the majority of neighboring objects. Ties are broken in an unspecified manner, for example, randomly or by taking the most frequent class in the training set.

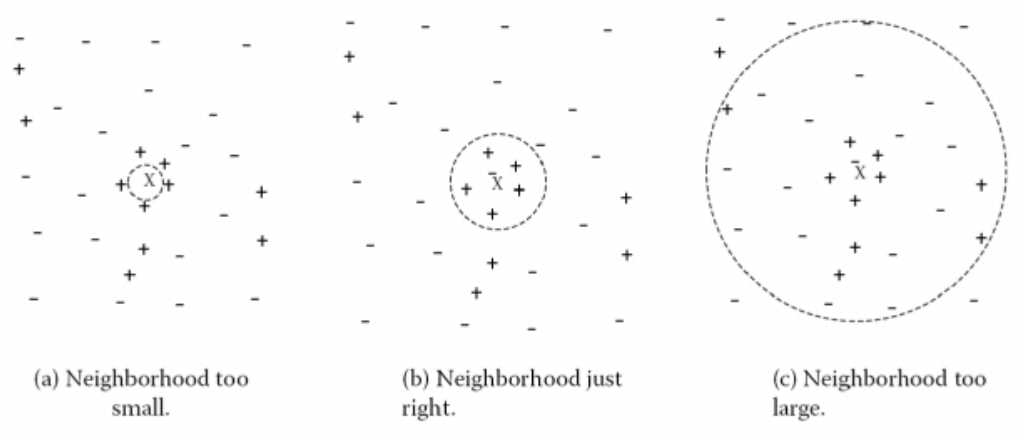

Figure 4.2: Choosing k value

Given an $m_{x}{ }^{*} n$ data matrix $\mathrm{X}$, which is treated as $m_{x} l *_{n}$ row vectors $x_{1,}, x_{2, \ldots}, x_{m x}$, and $m_{y}{ }^{*} n$ data matrix $\mathrm{Y}$, which is treated as $m_{y}{ }^{*} n$ row vectors $y_{1}, y_{2, \ldots}, y_{m y}$, the Euclidean distances between the vectors $x_{s}$ and $y_{t}$ are defined as follows:

$$
d_{s t}^{2}=\left(x_{s}-y_{t}\right)\left(x_{s}-y_{t}\right)^{\prime}
$$

Selection of $k$ value is very important in $\mathrm{kNN}$ classifier, this is explained in the Figure 4.2.

\section{SUPPORT VECTOR MACHINE}

SVM is a binary classifier, SVM classifies data by finding best hyperplane that separates all data points of one class from the other. The best hyperplane for an SVM means the one with the largest margin between the two classes.

The support vectors are the data points that are closest to the separating hyperplane, these points are on the boundary of the slab. Figure 4.3 illustrates these definitions, with + indicating data points of type 1 , and - indicating data points of type $-1[6,7]$. 


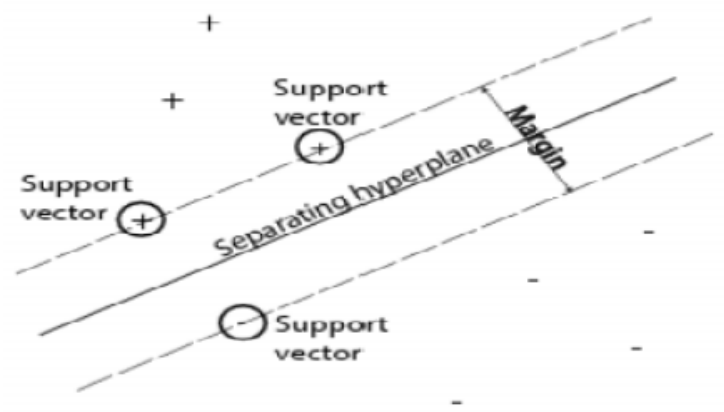

Figure 4.3: Working of SVM

The data for training is a set of points (vectors) $x_{i}$, along with their categories $y_{i}$. For some dimension $d$, the $\mathrm{n} x_{i} \in R^{d}$, and the $y_{i}=\mp 1$. The equation of a hyperplane is $\langle w, x\rangle+b=0$ where, $w \epsilon R^{d},\langle w, x\rangle$ is the inner dot product of $w$ and $b$ is real.

For a vector $z$ the optimal solution can be gives as

$$
\operatorname{class}(z)=\operatorname{sign}(<w, z>+b)
$$

The supervised data along with the kernel function is used to train SVM,

- The data input is a matrix, where each row is one observation and each column is one feature.

- The group to which the supervised data belongs is a column vector with each row corresponding to the value corresponding row in data.

- The kernel function and training parameters are changed for optimized performance.

For the quadratic kernel function with dimension, $d=9$ it can be given by

$$
k(x, y)=(1+<x, y>)^{d}
$$

\section{RESULTS}

Acquisition of the skin burn images is done by ordinary camera which can be found in basic mobile phones or a laptop web-cam and the images used are captured in usual ambiance without any particular lighting condition or isolation, this makes our work user-friendly. Images acquired in these conditions are noisy due to poor illumination, lack of dynamic range and wrong setting of the lens aperture and also may be due to environmental conditions. Hence enhancement of these images before categorizing into various grades is necessary and vital.

\section{A. DATABASE}

Our database consists of 120 images, 40 images from each grade are collected from various sources like internet, personally captured from Hospitals and scanned. All images are set to standard jpeg format and used in this work. 
Table 5.1: Database of Burn Images

\begin{tabular}{|l|c|c|c|c|}
\hline Type & $\begin{array}{l}\text { No. of } \\
\text { Images }\end{array}$ & Internet & Captured & $\begin{array}{l}\text { Scanned } \\
\text { form } \\
\text { books }\end{array}$ \\
\hline Grade1 & 40 & 8 & 27 & 5 \\
\hline Grade2 & 40 & 7 & 28 & 5 \\
\hline Grade3 & 40 & 4 & 32 & 4 \\
\hline
\end{tabular}

\section{B. IMAGE CLASSIFICATION}

Figure 6.1 shows the chrominance plot of features, mean and DCT values of 120 skin burn images belonging to 3 different categories. Features of 40 images belonging to each category are plotted. $(2,1)^{\text {th }}$ coefficient of DCT values are plotted on $\mathrm{x}$-axis and chrominance mean on y-axis. The distribution of these features allows the classifiers to learn and discriminate the test sample values into different category.

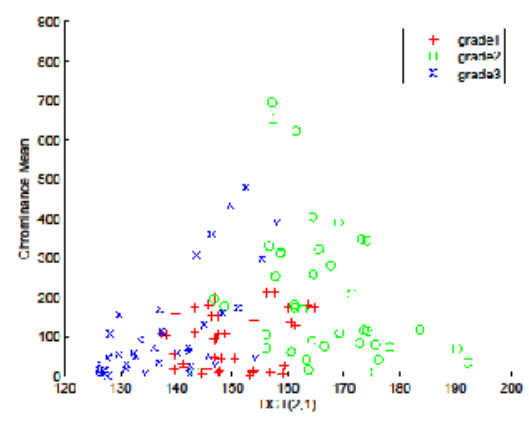

Figure 5.1: Feature Space

\section{TEMPLATE MATCHING METHOD}

In this method of classification, a new test vector containing mean and DCT values of sample images is matched with the previously stored templates belonging to different grades. Depending on the closeness of the match, test sample is categorized. Testing of 120 images, with 40 images from each category is carried out. 20 images from each category are used for training and remaining 20 for testing with cross validation. Overall a classifier efficiency of $66 \%$ is incurred with this method, the results are tabulated as shown in the Table 5.2.

Table 5.2: Template Matching Method

\begin{tabular}{|c|c|c|c|c|c|c|}
\hline \multirow{2}{*}{$\begin{array}{l}\text { Ty } \\
\text { pe } \\
\text { of } \\
\text { Im }\end{array}$} & \multirow{2}{*}{$\begin{array}{l}\text { No. } \\
\text { of } \\
\text { Im } \\
\text { age }\end{array}$} & \multirow{2}{*}{$\begin{array}{l}\text { Corr } \\
\text { ectly } \\
\text { Class } \\
\text { ified }\end{array}$} & \multicolumn{3}{|c|}{$\begin{array}{l}\text { Misclassified } \\
\text { as }\end{array}$} & \multirow{2}{*}{$\begin{array}{l}\text { Effi } \\
\text { cie } \\
\text { ncy } \\
\text { in }\end{array}$} \\
\hline & & & Gr & $\mathbf{G r}$ & $\mathbf{G r}$ & \\
\hline $\mathbf{G r}$ & 40 & 27 & & 4 & 9 & 67. \\
\hline Gr & 40 & 33 & 5 & & 2 & 82. \\
\hline $\mathbf{G r}$ & 40 & 30 & 7 & 3 & & 75 \\
\hline
\end{tabular}




\section{D. kNN Classifier}

In this method, classification is based on nearest neighbor method. Depending on the value of the $k$ specified, algorithm checks for the $\mathrm{k}$ nearest neighbors of training values, surrounding a test value. The majority of the test values determine the class of test value. In this work, multi class $\mathrm{kNN}$ classifier is used with $k$ value as 3 . Cross validation of training and testing vectors are carried out by using 3 fold techniques by randomly selecting training and testing vectors. This method has $75 \%$ of efficiency.

\section{E. SVM CLASSIFIER}

SVM is a binary classifier and the data are classified using support vectors. Separating hyperplane is created based on the kernel function. Complexity varies accordingly with the kernel function. In our case for multi classification, one against all property is used by choosing quadratic kernel function. Binary classification result is plotted with 3 fold cross validation. SVM classifier has resulted with an efficiency of $90 \%$ as shown in the below table.

Table 5.3: kNN Classifier

\begin{tabular}{|c|c|c|c|c|c|c|}
\hline \multirow{2}{*}{$\begin{array}{l}\text { Type of } \\
\text { Image }\end{array}$} & \multirow{2}{*}{$\begin{array}{l}\text { No. of } \\
\text { Images }\end{array}$} & \multirow{2}{*}{$\begin{array}{l}\text { Correctly } \\
\text { Classified }\end{array}$} & \multicolumn{3}{|c|}{ Misclassified as } & \multirow{2}{*}{$\begin{array}{l}\text { Efficiency in } \\
\%\end{array}$} \\
\hline & & & Grade1 & Grade2 & Grade3 & \\
\hline Grade1 & 40 & 22 & & 6 & 12 & 55 \\
\hline Grade2 & 40 & 29 & 4 & & 7 & 72.5 \\
\hline Grade3 & 40 & 28 & 9 & 3 & & 70 \\
\hline
\end{tabular}

Table 5.4: SVM Classifier

\begin{tabular}{|c|c|c|c|c|c|c|}
\hline & \multirow{2}{*}{$\begin{array}{ll}\text { No. } & \text { of } \\
\text { Images }\end{array}$} & \multirow{2}{*}{$\begin{array}{l}\text { Correctly } \\
\text { Classified }\end{array}$} & \multicolumn{3}{|c|}{ Misclassified as } & \multirow{2}{*}{$\begin{array}{l}\text { Efficienc } \\
\mathrm{y} \text { in \% }\end{array}$} \\
\hline & & & Grade1 & Grade2 & Grade3 & \\
\hline Grade1 & 40 & 34 & & 2 & 4 & 85 \\
\hline Grade2 & 40 & 35 & 3 & & 2 & 87.5 \\
\hline Grade3 & 40 & 37 & 2 & 1 & & 92.5 \\
\hline
\end{tabular}

Graph in the Figure 5.1 shows the binary classification results, using the one against all property for multi class classification. The graphs show training, classified and support vectors. Here it is observed that the line separating the image features belonging to different group, the points marked with the circles are the support vectors chosen by the kernel function for separation. 


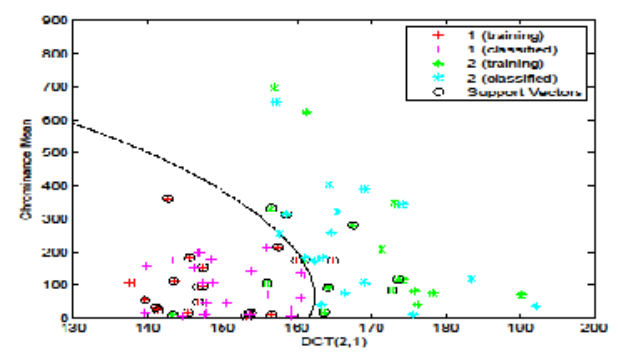

Figure 5.1: Classification of grade1 and grade2 vectors

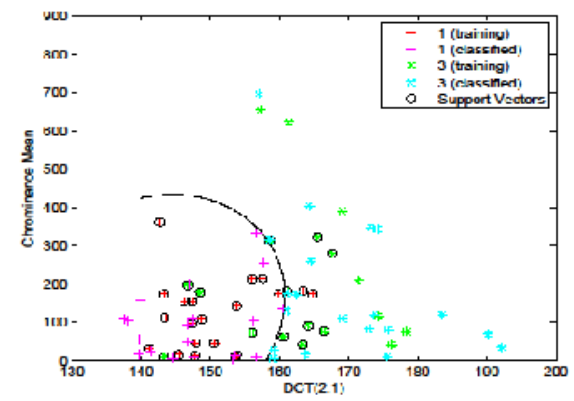

Figure 5.2: Classification of grade1 and grade3 vectors

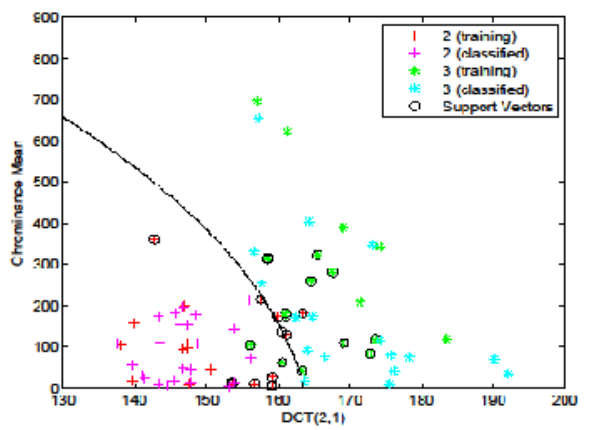

Figure 5.3: Classification of grade2 and grade 3 vectors

\section{CONCLUSION}

Examination of the classifier results shows, that the performance of the classifier has improved from Template Matching to kNN and further to SVM method. Performance degradation in Template Matching and kNN classifiers in comparison with SVM is due to the consideration of diverse database constructed from the images belonging to the people of different race, gender and age under differing ambient light unlike the references.

Here the features considered for the classification are simpler as mean and $(2,1)^{\text {th }}$ coefficient of DCT, which can be obtained easily with not much complex algorithms as cited in references. This reduces the complexity of calculations and thus the result computation time, making the implementation of the algorithm simpler and quick in decision taking for real time applications. The novelty of the work reported here is that it is simple, can be built using commercially available devices such as Camera and laptop. Using internet and mobile phones, the work can be converted to a tele- medicine project. The system could be made battery operated and thus deployed at remote places, lacking power grid connection. 


\section{ACKNOWLEDGMENT}

Authors are thankful to Sri Devraj Urs Medical Center for providing the burn wound photographs and Shrinivas the photographer who captured them, is appreciated and acknowledged. We would like to thank Dr. Kamal kumar, Plastic surgeon, Victoria hospital for his contributions.Also would like to thank Dr. Jyothi Subash, Dermatologist for her views on this topic.

\section{REFERENCES}

[1] B. Acha, C. Serrano, and L. Roa, "Segmentation and classification of burn color images," in Engineering in Medicine and Biology Society. Proceedings of the 23rd Annual Internal Conference of the IEEE, vol.3. pp. 2692-2695, 2001.

[2] B. Acha, C. Serrano, S. Palencia, and J. Murillo, "Classification of burn wounds using support vector machines," in Proceedings of SPIE, vol. 5370, p.1018, 2004.

[3] C. Serrano, L. Roa and B. Acha, "Evaluation of telemedicine platform in a burn unit," in Information Technology Applications in Biomedicene, of IEEE International Conference, pp. 121-126, 1998

[4] J.A. Clarke, "A color atlas of burn injuries," Chapman and Hall Medical, 1992.

[5] R. Gonzalez and R. Woods, "Digital image processing," IEEE Transactions on Systems, Man, and Cybernetics, 2008.

[6] R. Duda, P. Hart, and D. Stork, "Pattern classification, ed,2001.

[7] V. Vapnik, "Statistical learning theory," Wiley, New York, 1988.

[8] V. Vapnik, "Statistical Learning Theory". Wiley, New York, 1998

[9] Mathworks. (2012) SVM Classification.

\section{AUTHORS}

Malini Suvarna: Obtained her BE in Electronics andCommuniocation from Shri Jayachamrajendre College of Engineering, Mysore in 1997 and M.tech in Biomedical Engineering from Manipal Institute of Technology in 2002. At present working as Assistant Professor in E \& C Dept in Atria Institute of Technology, Bangalore.

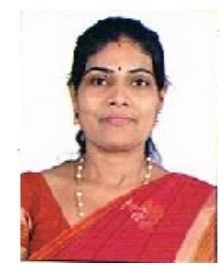

Dr. Niranjan U C: He obtained his Ph D in Electrical Science from Indian Institute of Science, Bangalore in 1993. He was a visiting Researcher at PARAMA Biomonitoring Institute, Fukuoka, Japan in 1994 and 1996. He is an Adjunct Professor at E\&C Department, MIT, Manipal and Director of Research \& Training at Manipal Dot Net, Manipal. He is the past Secretary of BMESI and a senior member of IEEE. He was the recipient of IEEE EMBS best student paper award in 1992 at Paris and Young Achiever paper award at ISCE, Arizona in 1996.

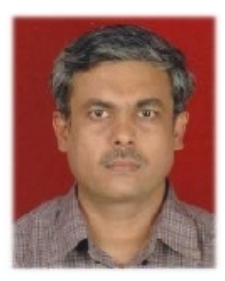

Dr.B.Sivakumar, M.E, PGDBA, Ph.D. Prof. B Sivakumar heads the Telecommunication Department in Dr.Ambedkar institute of Technology,Bangalore,Karnataka,india.. Prof. B Sivakumar was graduated from Madurai Kamaraj University in the field of Electronics and Communication. He also obtained his Masters degree from PSG College of Technology, Bharathiar University in the field of Applied Electronics. He has been awarded Doctoral Degree in the field of Information \& Communication Engineering from Anna University, Chennai. He has got

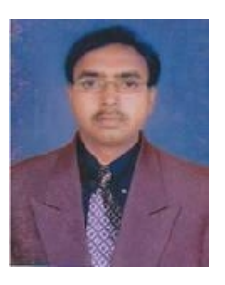
a rich teaching experience of 23 years. He has to his credit 20 International,7 National Journals and 40 Conference Papers. He is also Editor for an International Journal "ICTACT" and IJECE. Also reviewer for 5 International Journals. He is an recognized research guide for VTU and PRIST university as well as Doctoral Committee member for Anna University. He has delivered lot of expert lectures in the field of Wireless Communication. His interest of areas are Mobile communication, Genetic algorithm, Neural networks, Wireless ad-hoc networks, Image processing etc. 